\title{
SLIP EFFECTS ON BOUNDARY LAYER FLOW AND HEAT TRANSFER ALONG A STRETCHING CYLINDER
}

\author{
S. MUKHOPADHYAY \\ Department of Mathematics \\ The University of Burdwan \\ Burdwan-713104, W.B., INDIA \\ E-mail: swati_bumath@yahoo.co.in \\ R.S.R. GORLA* \\ Department of Mechanical Engineering \\ Cleveland State University \\ Cleveland, $\mathrm{OH} 44115$, USA \\ E-mail: r.gorla@csuohio.edu
}

\begin{abstract}
An axi-symmetric laminar boundary layer flow of a viscous incompressible fluid and heat transfer towards a stretching cylinder is presented. Velocity slip is considered instead of the no-slip condition at the boundary. Similarity transformations are used to convert the partial differential equations corresponding to the momentum and heat equations into non-linear ordinary differential equations. Numerical solutions of these equations are obtained by the shooting method. It is found that the velocity decreases with increasing the slip parameter. The skin friction as well as the heat transfer rate at the surface is larger for a cylinder compared to those for a flat plate.
\end{abstract}

Key words: boundary layer, stretching cylinder, partial slip, heat transfer, similarity solution.

\section{Introduction}

The study of a hydrodynamic flow and heat transfer over a stretching cylinder or flat plates has gained considerable attention due to its applications in industries and important bearings on several technological processes. Crane (1970) investigated the flow caused by the stretching of a sheet. Other researchers such as Gupta and Gupta (1977), Dutta et al. (1985), Chen and Char (1988) extended the work of Crane (1970) by considering the effects of heat and mass transfer analysis under different physical situations. Recently, various aspects of such problem have been investigated by many others such as Xu and Liao (2005), Cortell (2005; 2006), Hayat et al. (2006) and Hayat and Sajid (2007).

Lin and Shih $(1980 ; 1981)$ considered the laminar boundary layer and heat transfer along cylinders moving horizontally and vertically with constant velocity and found that the similarity solutions could not be obtained due to the curvature effect of the cylinder. Ishak and Nazar (2009) showed that similarity solutions might be obtained by assuming the cylinder stretching with linear velocity in the axial direction, claiming their study as an extension of the works by Grubka and Bobba (1985) and Ali (1994), from a stretching sheet to a stretching cylinder.

All of the authors mentioned above, continued their investigations by assuming the no slip boundary conditions. The non-adherence of the fluid to a solid boundary, known as velocity slip, is a phenomenon that has been observed under certain circumstances (Yoshimura and Prudhomme, 1998). Recently, many

\footnotetext{
* To whom correspondence should be addressed
} 
researchers viz. Wang (2002), Andersson (2002), Ariel et al. (2006), Ariel (2008), Abbas et al. (2009) etc. investigated the flow problems taking the slip flow condition at the boundary.

Since no attempt has ever been made to analyze the effects of partial slip on a boundary layer axisymmetric flow and heat transfer along a stretching cylinder, the same is considered in this paper. Hence, this work may be considered as an extension of the work of Ishak and Nazar (2009). Using similarity transformation, a third order ordinary differential equation corresponding to the momentum equation and a second order ordinary differential equation corresponding to heat equation are derived. Using the shooting method numerical calculations up to a desired level of accuracy are carried out for different values of the dimensionless parameters, so as to illustrate the results graphically. The results are compared with those of Ishak and Nazar (2009), Grubka and Bobba (1985) and Ali (1994). The analysis of the results shows that the flow field is influenced appreciably by the slip parameter. Estimations of the skin friction and heat transfer coefficient which are very important from the view point of industrial application, are also presented in the analysis.

\section{Equations of motion}

Consider the steady axi-symmetric flow of an incompressible viscous fluid along a stretching cylinder. The continuity, momentum and energy equations governing such type of flow are written as

$$
\begin{aligned}
& \frac{\partial(r u)}{\partial x}+\frac{\partial(r v)}{\partial r}=0 \\
& u \frac{\partial u}{\partial x}+v \frac{\partial u}{\partial r}=\frac{v}{r} \frac{\partial}{\partial r}\left(r \frac{\partial u}{\partial r}\right), \\
& u \frac{\partial T}{\partial x}+v \frac{\partial T}{\partial r}=\frac{\kappa}{r} \frac{\partial}{\partial r}\left(r \frac{\partial T}{\partial r}\right)
\end{aligned}
$$

where $u$ and $v$ are the components of velocity in the $x$ and $r$ directions respectively, $v=\frac{\mu}{\rho}$ is the kinematic viscosity, $\rho$ is the fluid density (assumed constant), $\mu$ is the coefficient of fluid viscosity, $\kappa$ is the thermal diffusivity of the fluid, $T$ is the fluid temperature.

The appropriate boundary conditions for the problem are given by

$$
\begin{aligned}
& u=U(x)+B_{l} v \frac{\partial u}{\partial r}, \quad v=0, \quad T=T_{w}(x) \quad \text { at } \quad r=R, \\
& u \rightarrow 0, \quad T \rightarrow T_{\infty} \quad \text { as } \quad r \rightarrow \infty .
\end{aligned}
$$

Here $U(x)=U_{0} \frac{x}{L}$ is the stretching velocity, $T_{w}(x)=T_{\infty}+T_{0}\left(\frac{x}{L}\right)^{N}$ is the prescribed surface temperature, $U_{0}, T_{0}$ are the reference velocity and temperature respectively, $T_{\infty}$ is the ambient temperature, $L$ is the characteristic length, $N$ is the temperature exponent, $B_{1}$ is the velocity slip. 


\subsection{Method of solution}

The continuity equation is automatically satisfied by the introduction of the stream function $\psi$ as

$$
u=\frac{1}{r} \frac{\partial \psi}{\partial r}, \quad v=-\frac{1}{r} \frac{\partial \psi}{\partial x}
$$

Introducing the similarity variables as

$$
\eta=\frac{r^{2}-R^{2}}{2 R}\left(\frac{U}{v x}\right)^{\frac{1}{2}}, \quad \psi=(U v x)^{\frac{1}{2}} R f(\eta), \quad \theta(\eta)=\frac{T-T_{\infty}}{T_{w}-T_{\infty}}
$$

and substituting Eqs (2.6) in Eqs (2.2), (2.3), (2.4) and (2.5), the governing equations and the boundary conditions reduce to

$$
\begin{aligned}
& (1+2 M \eta) f^{\prime \prime \prime}+2 M f^{\prime \prime}+f f^{\prime \prime}-f^{\prime 2}=0, \\
& (1+2 M \eta) \theta^{\prime \prime}+2 M \theta^{\prime}+\operatorname{Pr}\left(f \theta^{\prime}-N f^{\prime} \theta\right)=0, \\
& f^{\prime}=1+B f^{\prime \prime}, \quad f=0, \quad \theta=1 \quad \text { at } \quad \eta=0,
\end{aligned}
$$

and

$$
f^{\prime} \rightarrow 0, \quad \theta^{\prime} \rightarrow 0 \quad \text { as } \quad \eta \rightarrow \infty
$$

where the prime denotes differentiation with respect to $\eta, B=B_{1} \sqrt{\frac{U_{0} v}{L}}$ is the slip parameter and $M=\left(\frac{v L}{U_{0} R^{2}}\right)^{\frac{1}{2}}$ is the curvature parameter. The no-slip case is recovered for $B=0$. One can note that if $M=0$ (i.e., $R \rightarrow \infty$ ), the problem under consideration (with $B=0$ ) reduces to the boundary layer flow along a stretching flat plate considered by Ali (1994), with $m=1$ in that paper. Moreover, when $M=0$ (stretching flat plate) with $B=0$ (no-slip boundary condition), the analytical solutions of Eqs (2.7) and (2.8) were given by Crane (1970), Grubka and Bobba (1985), respectively.

\section{Numerical methods}

Equations (2.7)-(2.8) along with boundary conditions (2.9)-(2.10) are solved by converting them to an initial value problem. We set

$$
\begin{aligned}
& f^{\prime}=z, \quad z^{\prime}=p, \quad p^{\prime}=\left[z^{2}-f p-2 M p\right] /(1+2 M \eta), \\
& \theta^{\prime}=q, \quad q^{\prime}=-[\operatorname{Pr}(f q-N z \theta)+2 M q] /(1+2 M \eta),
\end{aligned}
$$

with the boundary conditions 


$$
f(0)=0, \quad f^{\prime}(0)=1+B \gamma, \quad f^{\prime \prime}(0)=\gamma, \quad \theta(0)=1 .
$$

In order to integrate (3.1) and (3.2) as initial value problems, one requires a value for $p(0)$, i.e., $f^{\prime \prime}(0)$ and a value for $q(0)$ i.e., $\theta^{\prime}(0)$ but no such values are given at the boundary. The suitable guess values for $f^{\prime /}(0)$ and $\theta^{\prime}(\theta)$ are chosen and then integration is carried out. Comparing the calculated values for $f^{\prime}$ and $\theta$ at $\eta=10$ (say) with the given boundary conditions $f^{\prime}(10)=0$ and $\theta(10)=0$ and adjusting the estimated values, $f^{\prime \prime}(0)$ and $\theta^{\prime}(0)$, a better approximation for the solution is obtained.

Taking the series of values for $f^{\prime \prime}(0)$ and $\theta^{\prime}(0)$ and applying the fourth order classical RungeKutta method with step-size $h=0.01$, the above procedure is repeated to achieve the desired degree of accuracy $\left(10^{-5}\right)$.

\section{Results and discussions}

Using the method described in the previous section, numerical computations for various values of the curvature parameter $(M)$, velocity slip parameter $(B)$, temperature exponent $(N)$ and Prandtl number $(\operatorname{Pr})$ have been carried out in order to analyse the results. To illustrate the results, numerical values are plotted in Figs $1 \mathrm{a}$ to 6 b.

For verification of accuracy of the applied numerical scheme, a comparison of the present results is made corresponding to the heat transfer coefficient $\left[\theta^{\prime}(0)\right]$ for $B=0$ (i.e., in the absence of slip) and $M=0$ (i.e., for a stretching flat plate) with the published results of Ishak and Nazar (2009), Grubka and Bobba (1985), Ali (1994) and presented in Tab.1. The results are found in excellent agreement.

Table 1. Values of $\mathrm{Nu}_{x} \mathrm{Re}_{x}^{-1 / 2}=-\theta^{\prime}(O)$ for several values of temperature exponent $N$ for flat plate $(M=0)$ with no-slip $(B=0)$ and $\operatorname{Pr}=1$.

\begin{tabular}{|c|c|c|c|c|}
\hline$N$ & Ishak and Nazar (2009) & Grubka and Bobba (1985) & Ali (1994) & Present study \\
\hline 0 & 0.5820 & 0.5820 & 0.5801 & 0.5821 \\
1 & 1.0000 & 1.0000 & 0.9961 & 1.0000 \\
2 & 1.3333 & 1.3333 & 1.3269 & 1.3332 \\
\hline
\end{tabular}

Let us first consider the effects of the curvature parameter $M$ on velocity distribution in cases of noslip and slip at the boundary. In Fig.1a, horizontal velocity profiles are shown for different values of $M$. The horizontal velocity curves show that the rate of transport decreases with increasing distance $(\eta)$ of the sheet. In all cases, the velocity vanishes at some large distance from the sheet (at $\eta=10$ ). The velocity increases with increasing values of $M$. Figure $1 \mathrm{~b}$ represents the velocity profiles for various values of the curvature parameter $M$ in the presence of slip at the boundary. Same result is noted. The velocity gradient at the surface is larger for a larger values of $M$, which produces a larger skin friction coefficient. 


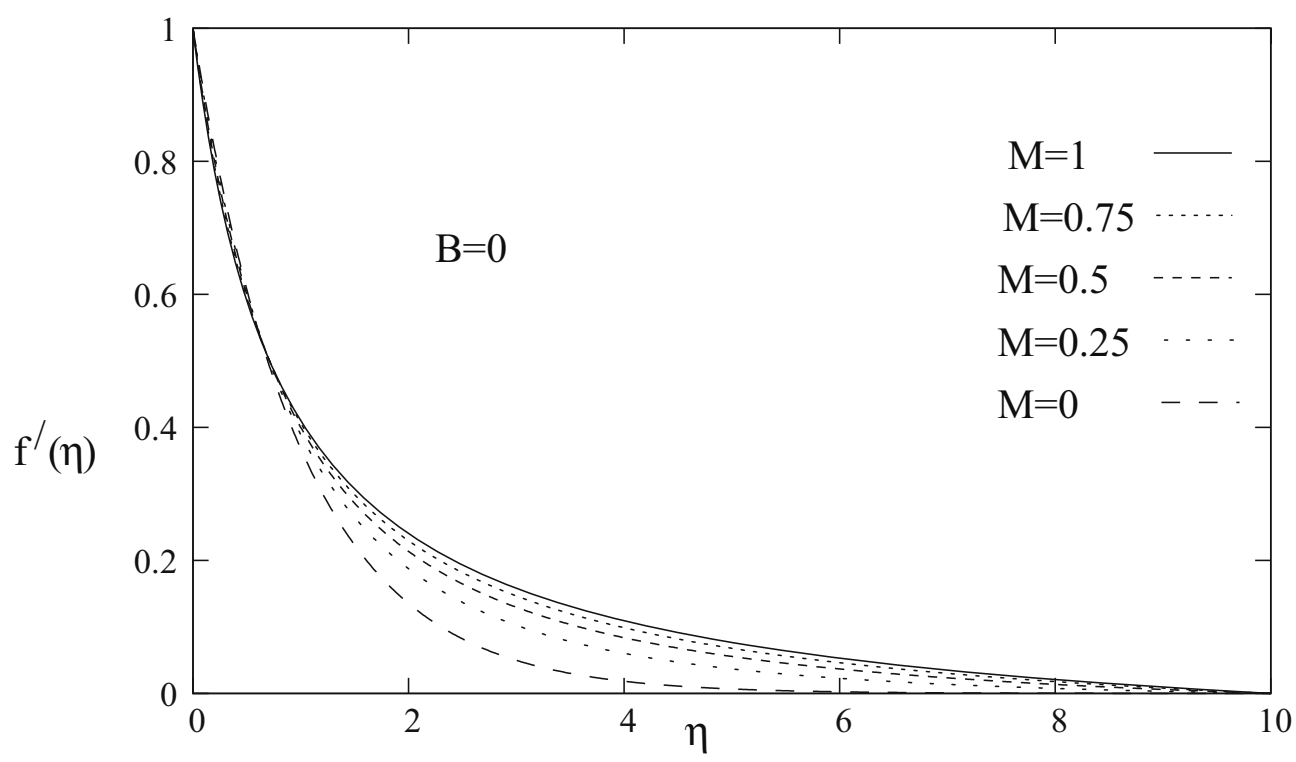

Fig.1a. Variation of velocity $f^{\prime}(\eta)$ with $\eta$ for several values of curvature parameter $M$ of the stretching cylinder in the absence of slip.

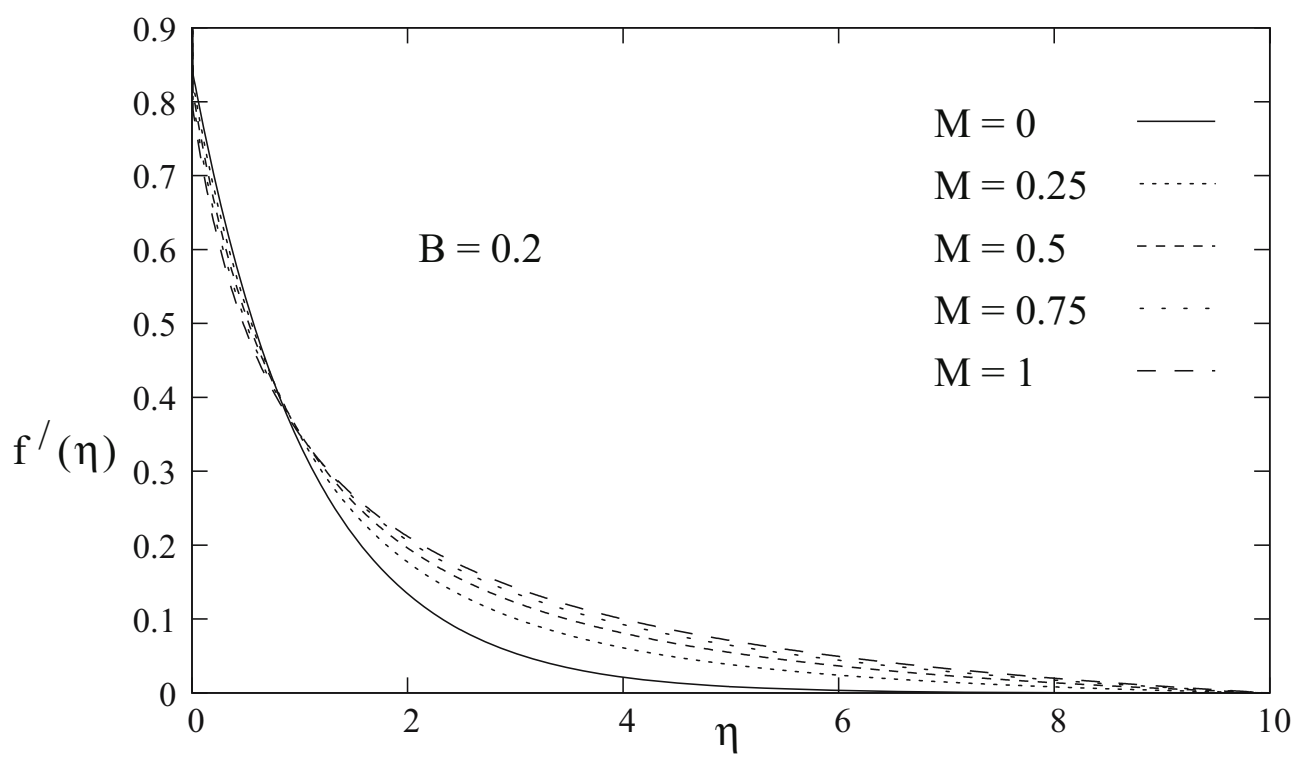

Fig.1b. Variation of velocity $f^{\prime}(\eta)$ with $\eta$ for several values of curvature parameter $M$ of the stretching cylinder in presence of slip.

Effects of the curvature parameter on the temperature distribution for no-slip and slip cases are presented in Fig.2a and Fig.2b respectively. In both cases, temperature is found to decrease with increasing the curvature parameter $M$. The thermal boundary layer thickness decreases as $M$ increases, which implies an increase in the wall temperature gradient and this in turn increases the surface heat transfer rate. Hence, the Nusselt number increases as $M$ increases. 


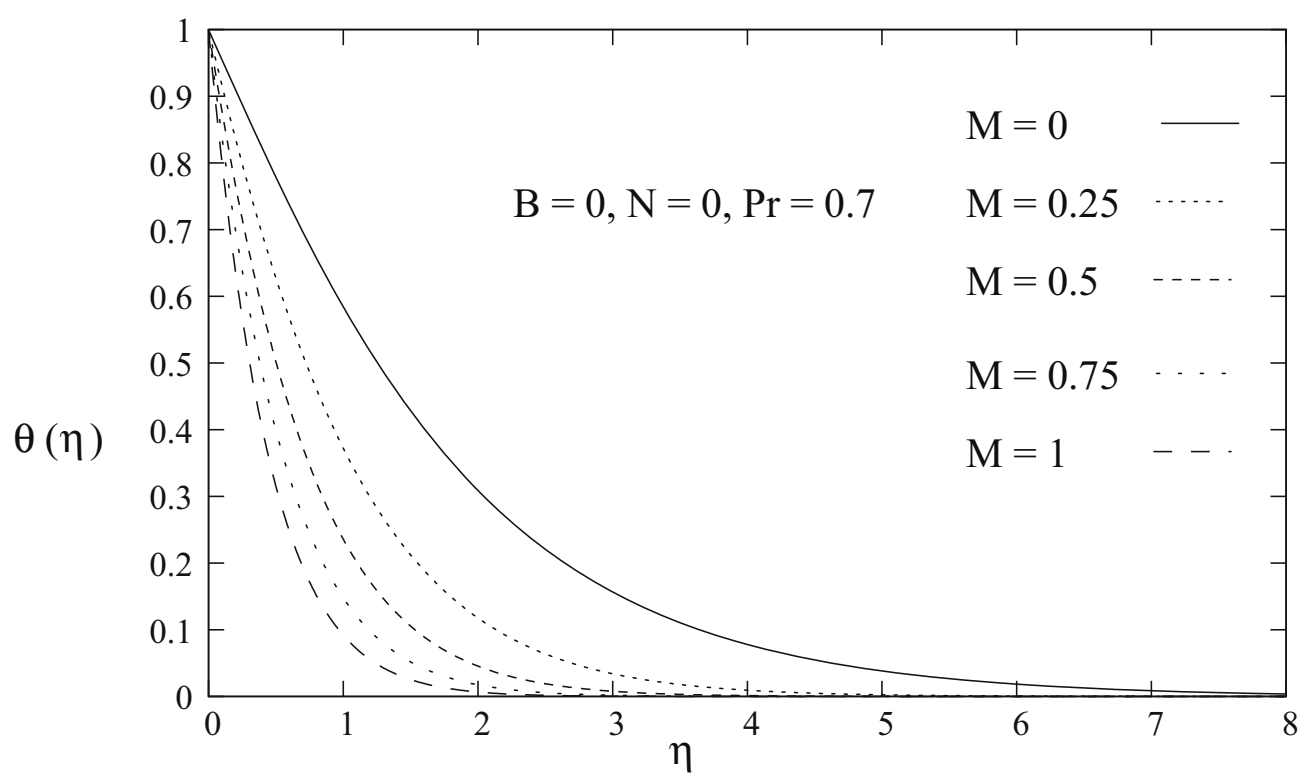

Fig.2a. Variation of temperature $\theta(\eta)$ with $\eta$ for several values of curvature parameter $M$ of the stretching cylinder in the absence of slip.

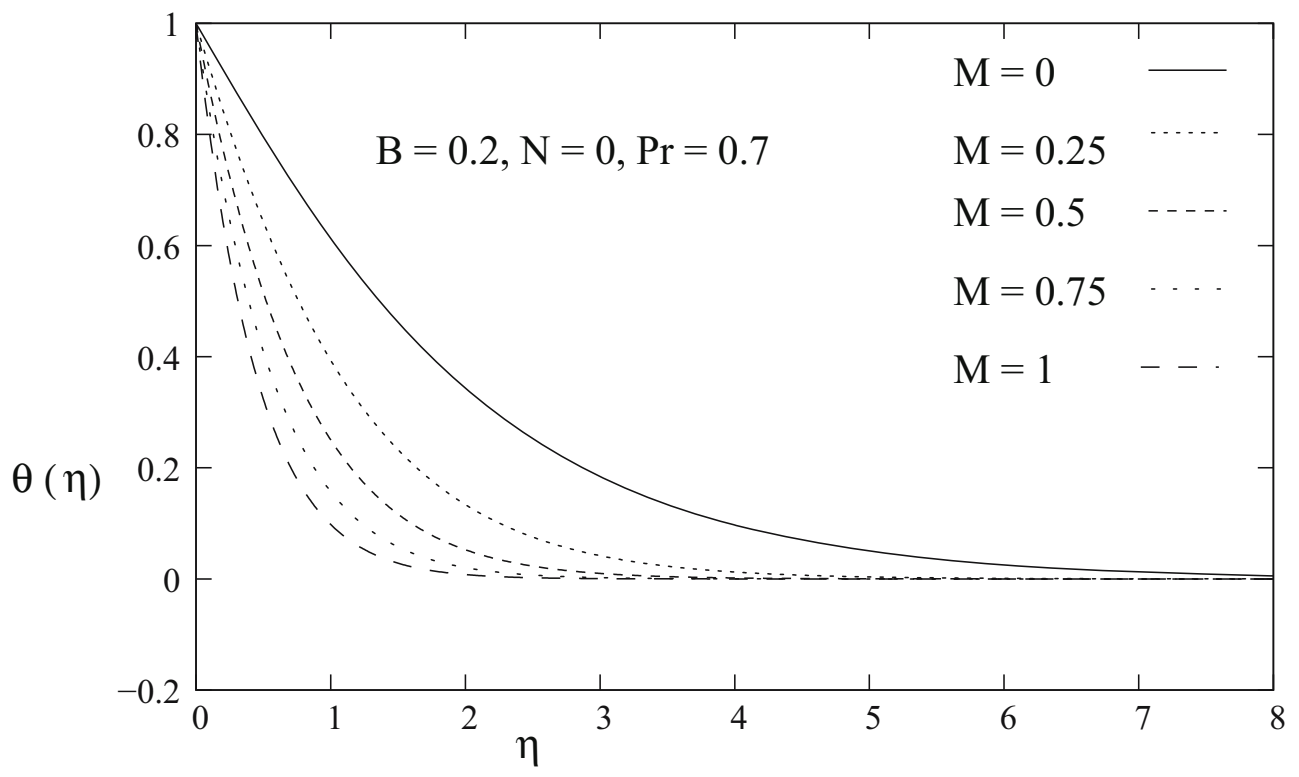

Fig.2b. Variation of temperature $\theta(\eta)$ with $\eta$ for several values of curvature parameter $M$ of the stretching cylinder in presence of slip.

Now, the velocity and shear stress profiles are presented for the variation of the velocity slip parameter for a flat plate and stretching cylinder respectively. Figure $3 \mathrm{a}$ and Fig. $3 \mathrm{~b}$ demonstrate the effects of the velocity slip parameter $(B)$ for a stretching flat plate (i.e., for $M=0$ ) on velocity and shear stress profiles, respectively. With increasing $B$, the horizontal velocity is found to decrease (Fig.3a). This decrease goes on up to certain heights and then the process is slowed down. When slip occurs, the flow velocity near the stretching wall is no longer equal to the stretching velocity of the wall. With the increase in $B$, such slip velocity increases and consequently fluid velocity decreases. This happens because the pulling of the stretching wall can be partly transmitted to the fluid under the slip condition. It is noted that $B$ has a 
substantial effect on the solutions. Figure $3 b$ exhibits that the shear stress $f^{\prime \prime}(\eta)$ increases with the increasing values of the velocity slip parameter $B . B=0$ corresponds to the no slip case. From the figure it is very clear that shear stress at the wall is negative here. Physically, a negative sign of $f^{\prime \prime}(0)$ implies that surface exerts a dragging force on the fluid and a positive sign implies the opposite. This is reasonable, since a stretching cylinder which induces the flow is considered here. Figure $3 \mathrm{c}$ presents the effects of slip the parameter $B$ on the stretching cylinder $(M=1)$. Here also velocity decreases but the temperature increases with increasing $B$ (Fig.4).

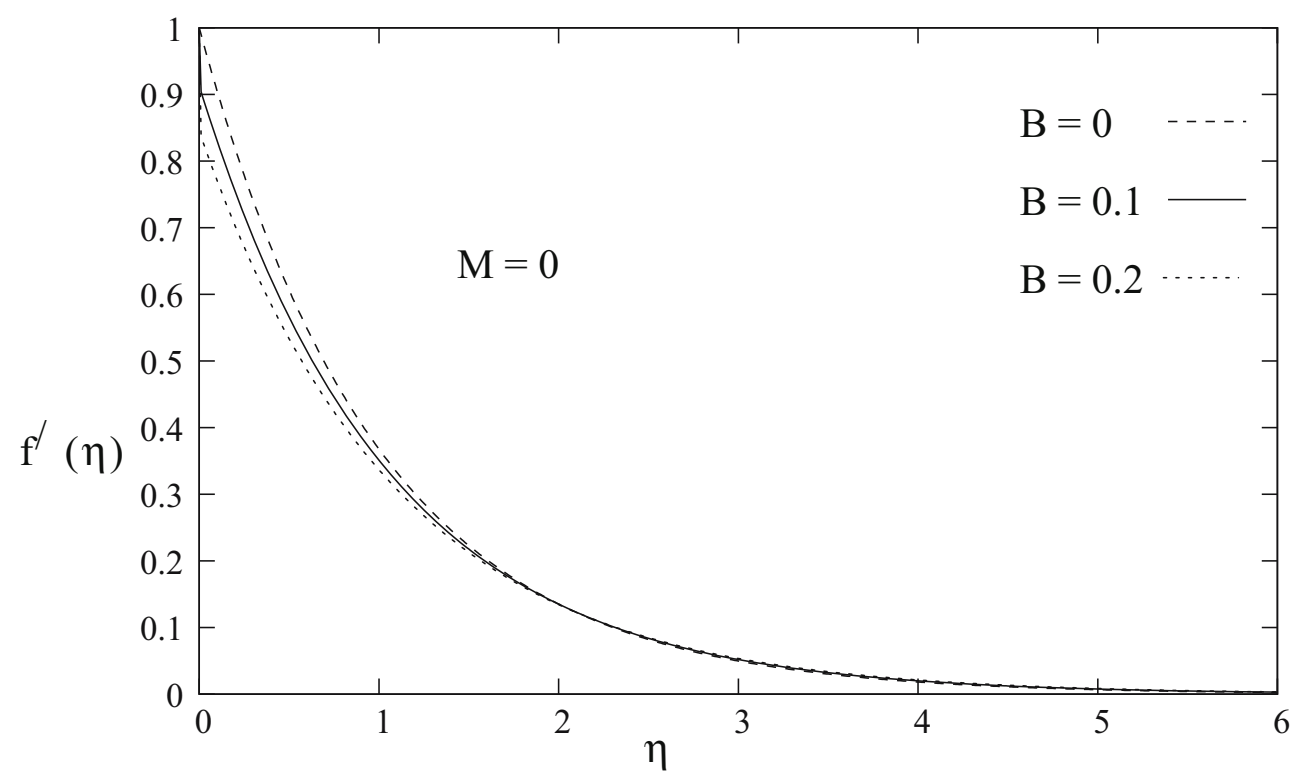

Fig.3a. Variation of velocity $f^{\prime}(\eta)$ with $\eta$ for several values of slip parameter $B$ for flat plate with $M=0$.

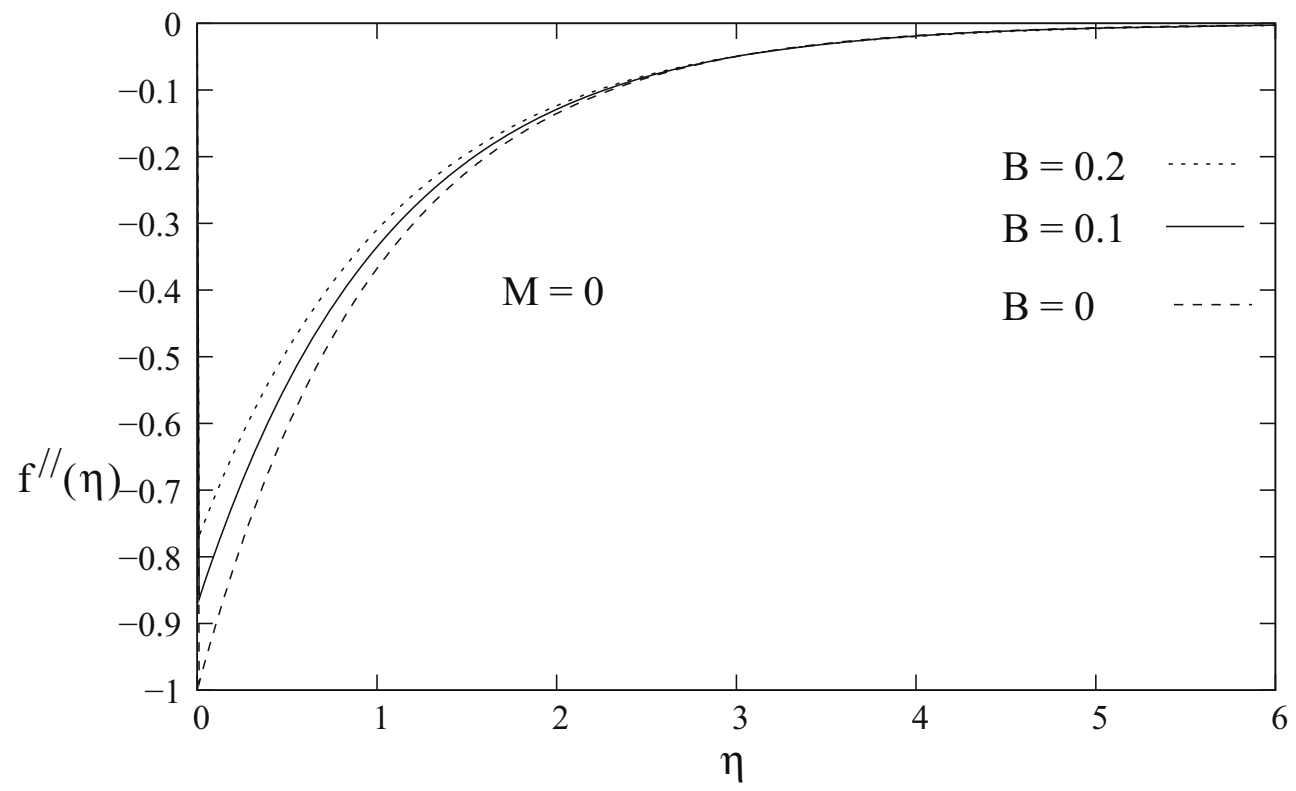

Fig.3b. Variation of shear stress $f^{\prime \prime}(\eta)$ with $\eta$ for several values of slip parameter $B$ for flat plate. 


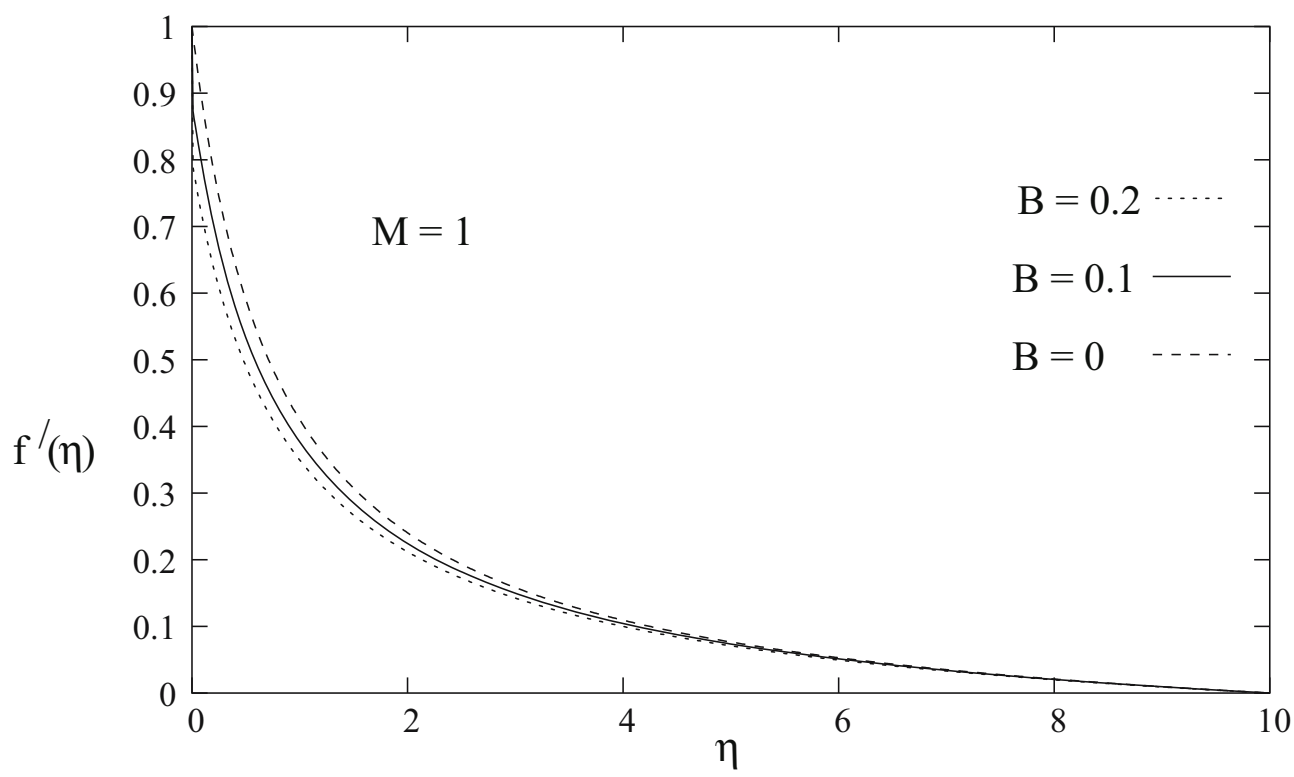

Fig.3c. Variation of velocity $f^{\prime}(\eta)$ with $\eta$ for several values of slip parameter $B$ for stretching cylinder.

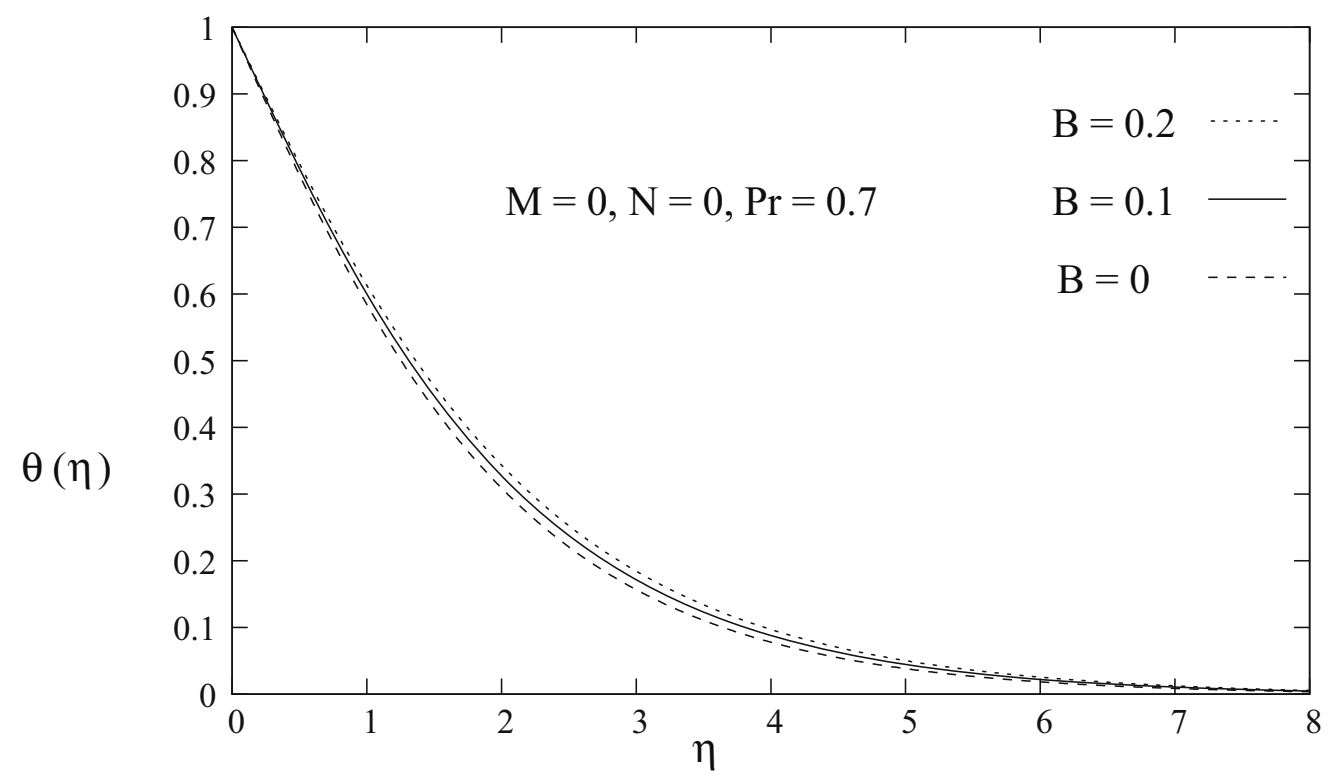

Fig.4. Variation of temperature $\theta(\eta)$ with $\eta$ for several values of slip parameter $B$ for flat plate.

Figure 5a and Fig.5b represent the temperature and temperature gradient profiles for the variable temperature exponent $N$ for a stretching flat plate $(M=0)$ in the presence of slip. It is seen that temperature increases with increasing values of $N$ and temperature overshoot is noted near the plate for $N>0$. The temperature gradient initially increases with $N$ but after a certain distance $\eta$ from the wall it decreases. Far away from the wall, such feature is smeared out. It is observed that for higher positive values of $N(=2,3)$, $\theta^{\prime}(\eta)$ is positive initially but finally it becomes negative. Temperature increases with $N$ but no temperature 
overshoot is noted for the stretching cylinder $(M=1)$ (Fig.5c). In the case of a stretching cylinder, the temperature gradient is negative for all values of $N$ (Fig.5d).

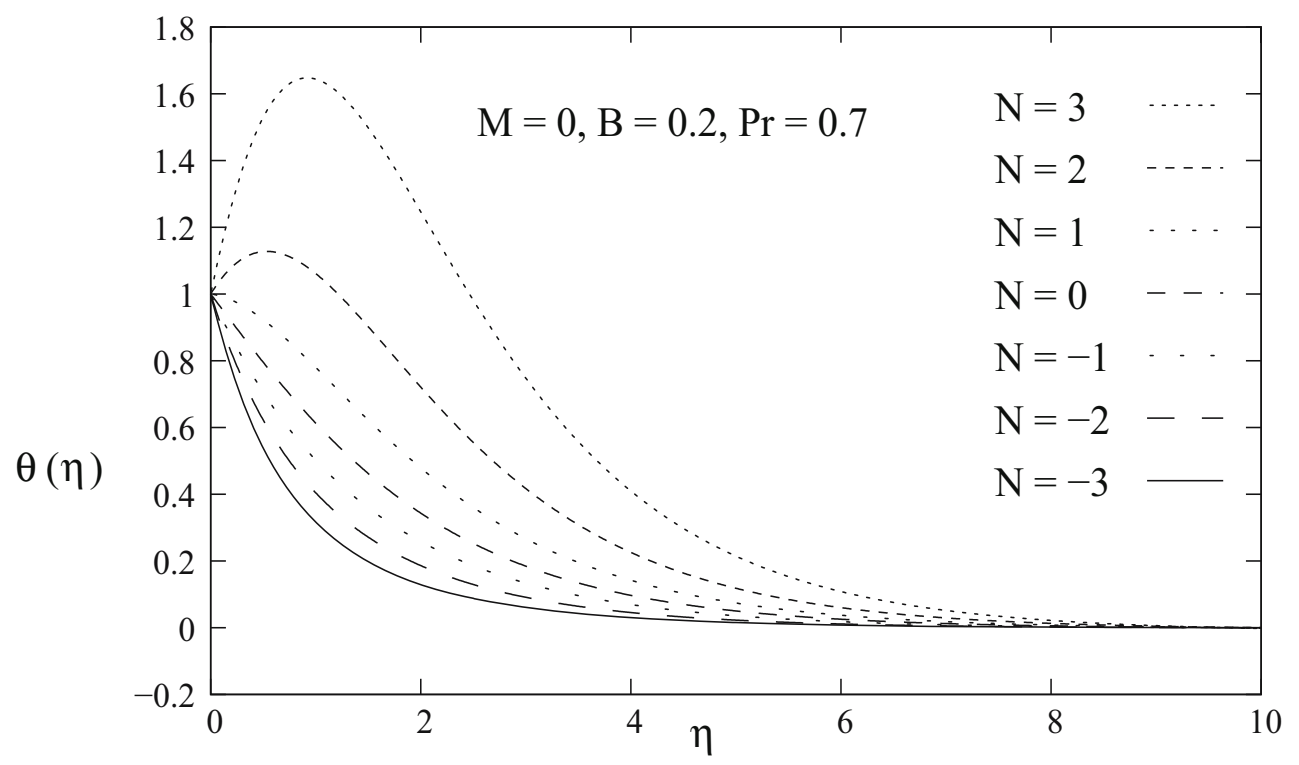

Fig.5a. Variation of temperature $\theta(\eta)$ with $\eta$ for several values of temperature exponent $N$ for flat plate.

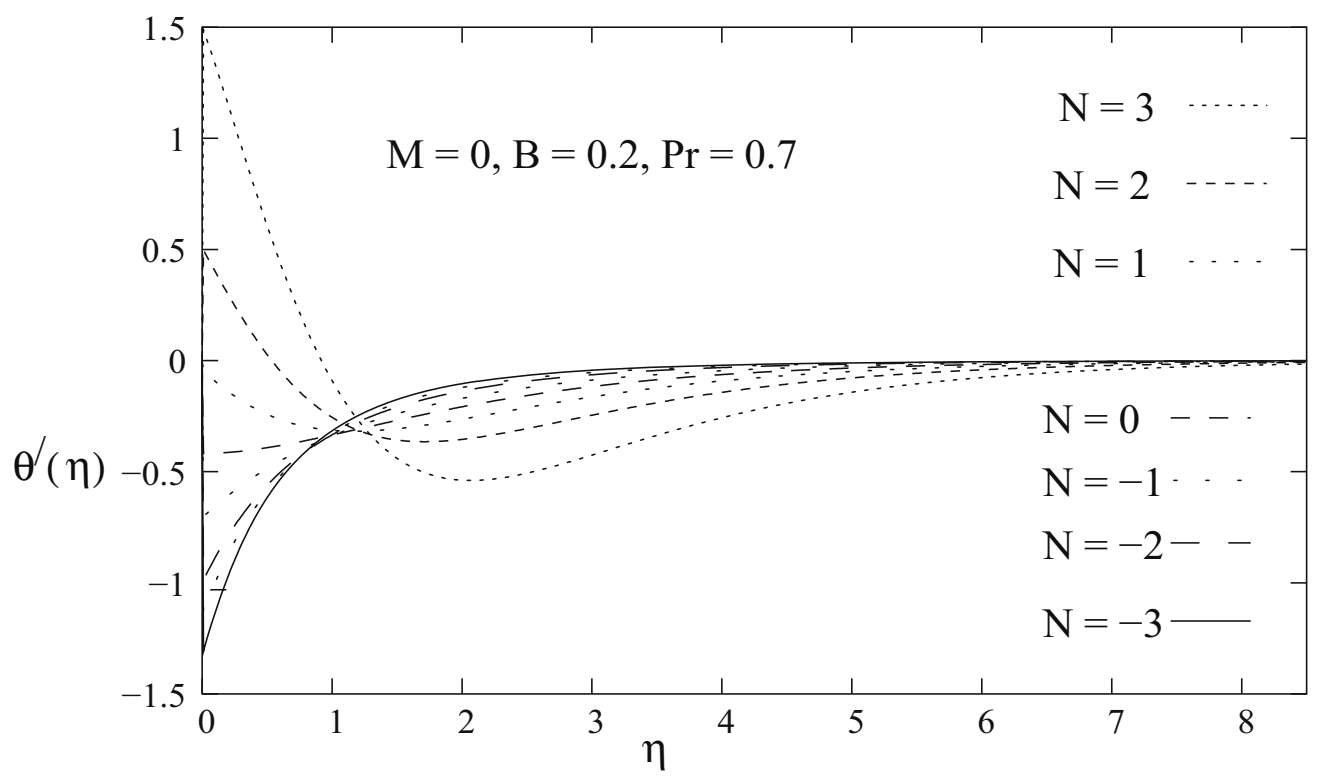

Fig.5b. Variation of temperature gradient $\theta^{\prime}(\eta)$ with $\eta$ for several values of temperature exponent $N$ for flat plate. 


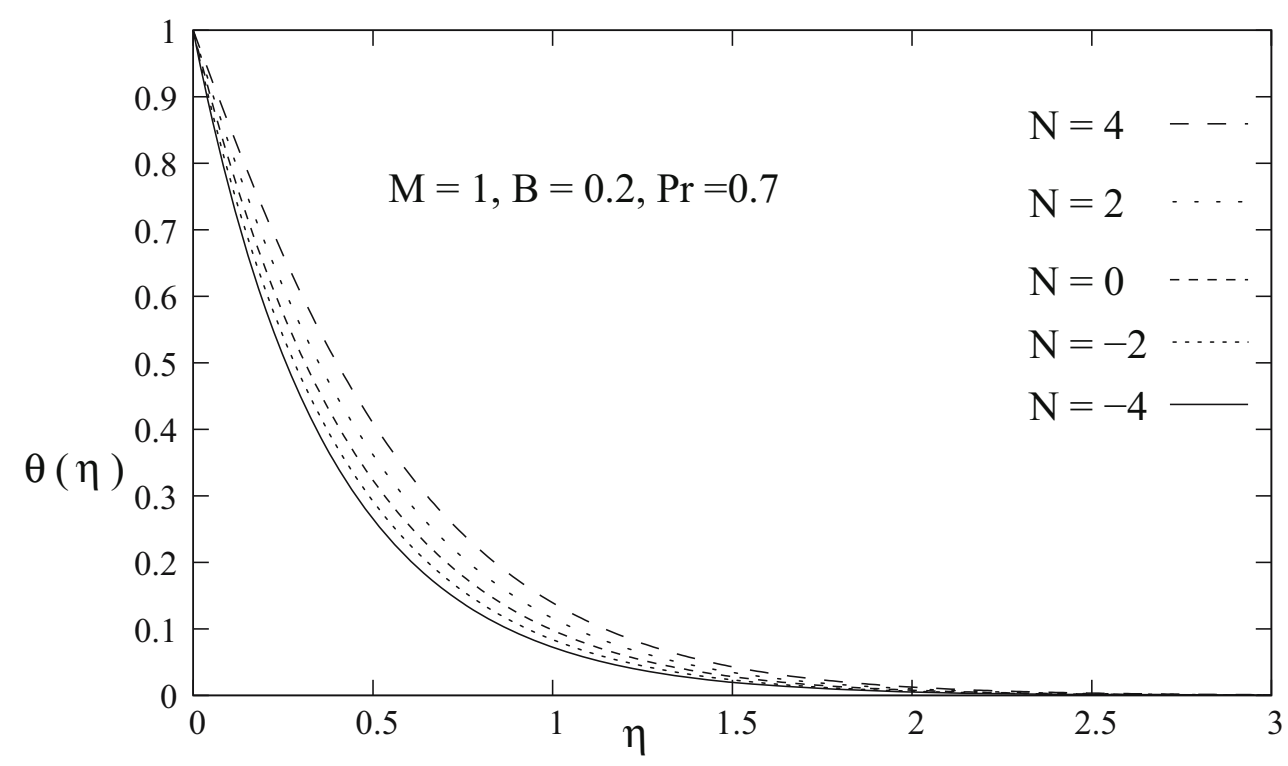

Fig.5c. Variation of temperature $\theta(\eta)$ with $\eta$ for several values of temperature exponent $N$ for stretching cylinder.

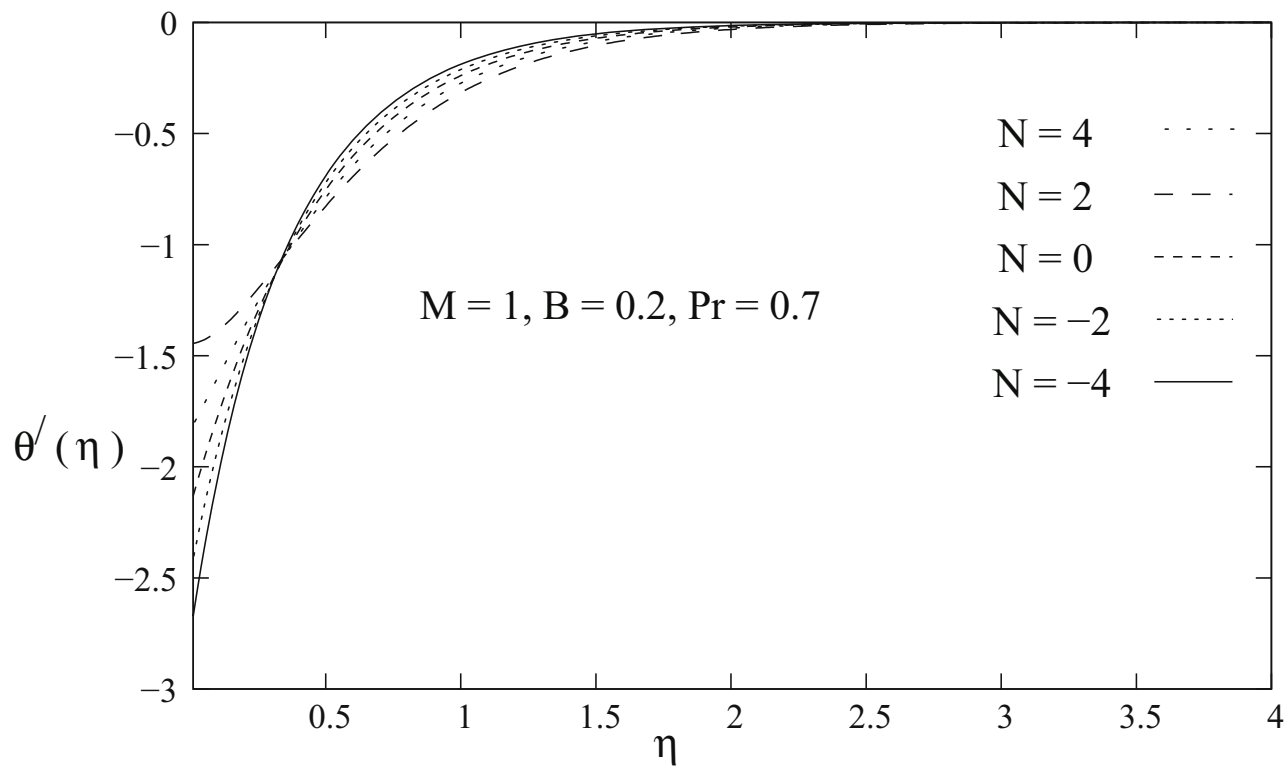

Fig.5d. Variation of temperature gradient $\theta^{\prime}(\eta)$ with $\eta$ for several values of temperature exponent $N$ for stretching cylinder.

Figure 6a exhibits the effect of the Prandtl number (Pr) on temperature profiles for a flat plate (i.e., for $M=0$ ) with $N=0$ in the presence of slip. Temperature is found to decrease with increasing Pr. An increase in the Prandtl number reduces the thermal boundary layer thickness. The Prandtl number signifies the ratio of momentum diffusivity to thermal diffusivity. Fluids with a lower Prandtl number will possess higher thermal conductivities (and thicker thermal boundary layer structures) so that heat can diffuse from the wall faster than that of fluids with a higher $\operatorname{Pr}$ (thinner boundary layers). Hence the Prandtl number may be used to increase the rate of cooling. 
Figure $6 \mathrm{~b}$ presents the behaviour of thermal field with increasing $\operatorname{Pr}$ for the stretching cylinder $(M=1)$ with $N=1$ in the presence of slip. It is very clear that the effects of $\operatorname{Pr}$ are much more prominent for a flat plate compared to that of a stretching cylinder.

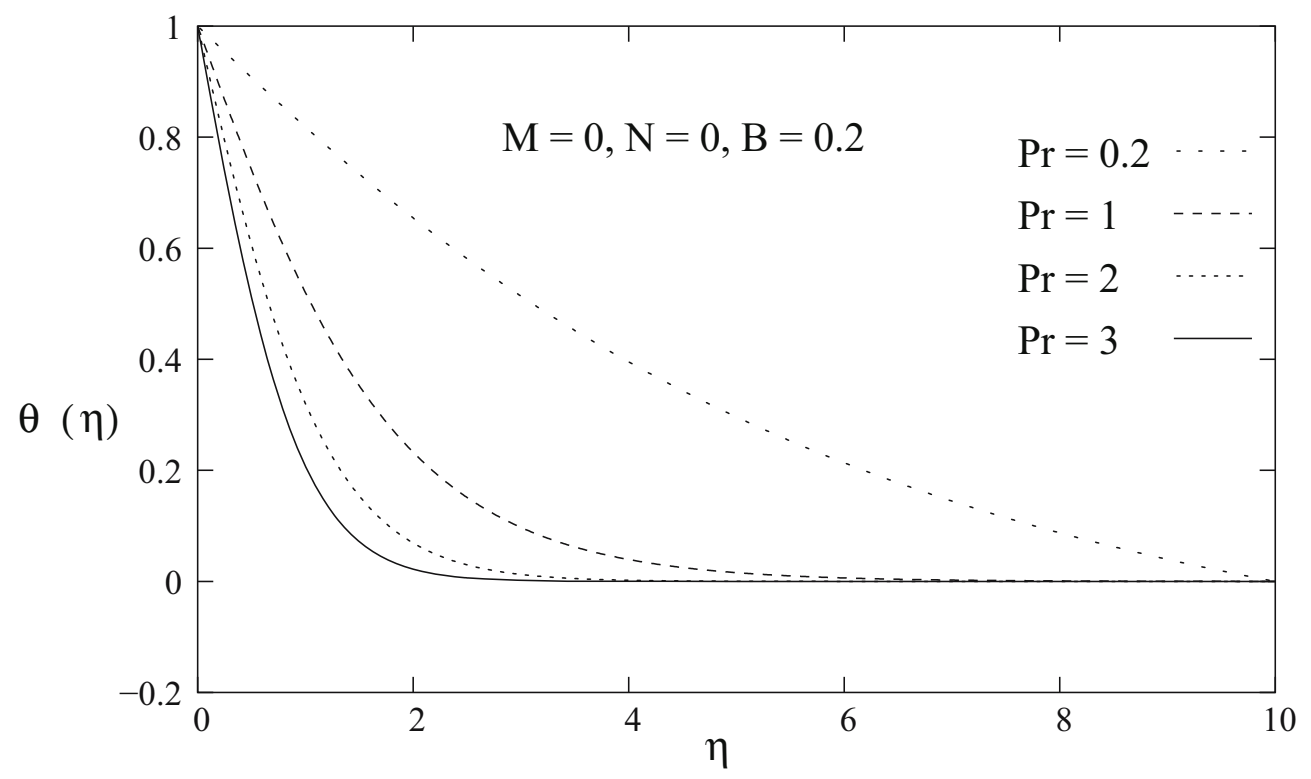

Fig.6a. Variation of temperature $\theta(\eta)$ with $\eta$ for several values of Prandtl number Pr for flat plate.

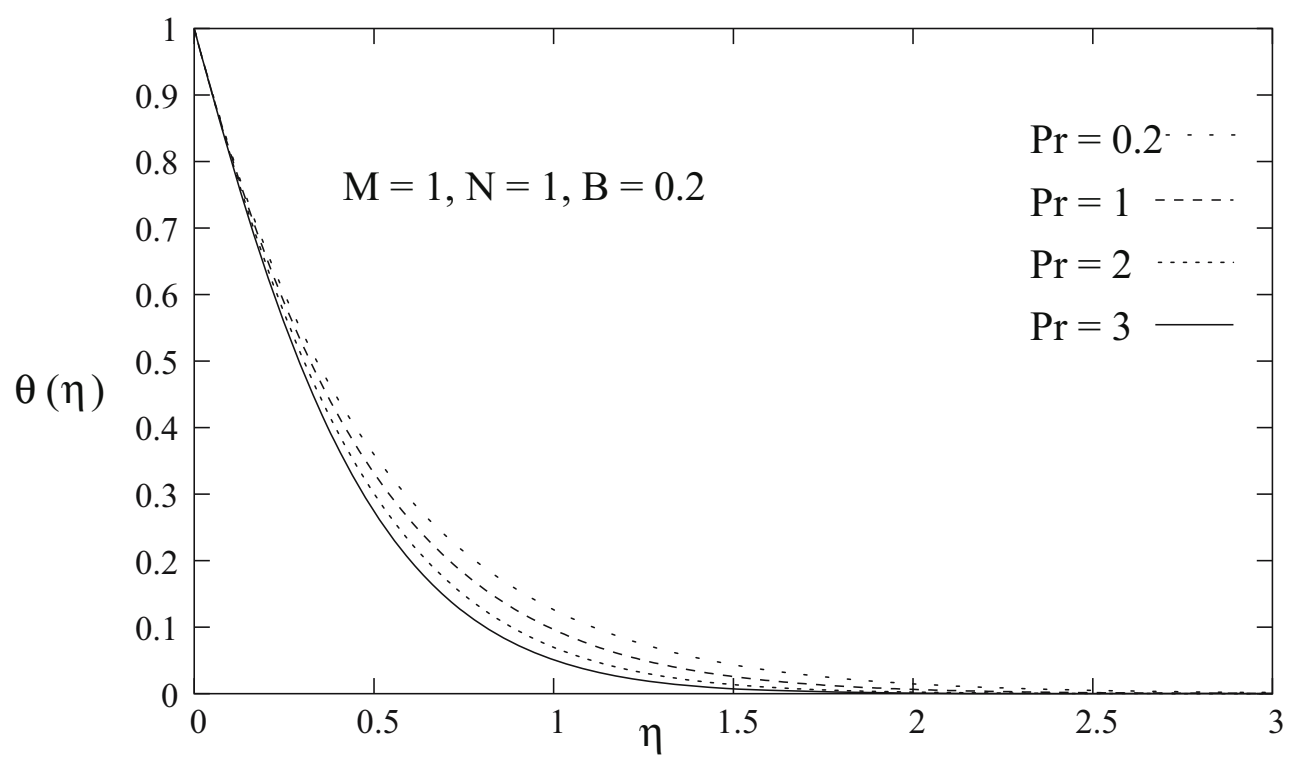

Fig.6b. Variation of temperature $\theta(\eta)$ with $\eta$ for several values of Prandtl number Pr for stretching cylinder.

\section{Nomenclature}

$$
\begin{aligned}
B & - \text { slip parameter } \\
f & - \text { dimensionless velocity function } \\
L & - \text { characteristic length } \\
M & - \text { curvature parameter }
\end{aligned}
$$


$N$ - exponent for surface temperature variation

Pr - Prandtl number

$R$ - radius of the cylinder

$r$ - radial direction

$T$ - temperature of the fluid

$T_{w}$ - surface temperature

$T_{\infty}$ - free-stream temperature

$U$ - stretching velocity

$u, v$ - components of velocity in $x$ and $r$ directions

$x$ - streamwise direction

$\eta-$ similarity variable

$\theta$ - non-dimensional temperature

$\kappa-$ thermal diffusivity

$\mu-$ dynamic viscosity

$v$ - kinematic viscosity

$\rho-$ density of the fluid

$\psi-$ stream function

\section{Subscripts}

$w$ - wall conditions

$\infty-$ conditions far away from the surface

\section{References}

Abbas Z., Wang Y., Hayat T. and Oberlack M. (2009): Slip effects and heat transfer analysis in a viscous fluid over an oscillatory stretching surface. - Int. J. of Numer. Meth. Fluids, vol.59, pp.443-458.

Ali M.E. (1994): Heat transfer characteristics of a continuous stretching surface. - Heat Mass Transfer, vol.29, pp.227234.

Andersson H.I. (2002): Slip flow past a stretching surface. - Acta Mech., vol.158, pp.121-125.

Ariel P.D. (2008): Two dimensional stagnation point flow of an elastico-viscous fluid with partial slip. - Z. Angew. Math. Mech., vol.88, pp.320-324.

Ariel P.D., Hayat T. and Asghar S. (2006): The flow of an elastico-viscous fluid past a stretching sheet with partial slip. - Acta Mech., vol.187, pp.29-35.

Chen C.K. and Char M.I. (1988): Heat transfer of a continuous stretching surface with suction or blowing. - J. Math Anal. Appl., vol.135, pp.568-580.

Cortell R. (2005): Flow and heat transfer of a fluid through a porous medium over a stretching surface with internal heat generation/absorption and suction/blowing. - Fluid Dynamics Resc., vol.37, pp.231-245.

Cortell R. (2006): Effects of viscous dissipation and work done by deformation on the MHD flow and heat transfer of a viscoelastic fluid oever a stretching sheet. - Physics Letters A, vol.357, pp.298-305.

Crane L.J. (1970): Flow past a stretching plate. - Z. Angew Math. Phys., vol.21, pp.645-647.

Datta B.K., Roy P. and Gupta A.S. (1985): Temperature field in the flow over a stretching sheet with uniform heat flux. - Int. Comm. Heat Mass Transfer, vol.12, pp.89-94.

Grubka L.G. and Bobba K.M. (1985): Heat transfer characteristics of a continuous stretching surface with variable temperature. - ASME J. Heat Transfer, vol.107, pp.248-250.

Gupta P.S. and Gupta A.S. (1977): Heat and mass transfer on a stretching sheet with suction or blowing. - Can. J. Chem. Eng., vol.55, pp.744-746. 
Hayat T., Abbas Z. and Sajid M. (2006): Series solution for the upper-convected Maxwell fluid over a porous stretching plate. - Physics Letters A, vol.358, pp.396-403.

Hayat T. and Sajid M. (2007): Analytic solution for axi-symmetric flow and heat transfer of a second grade fluid past a stretching sheet. - Int. J. of Heat and Mass Transfer, vol.50, pp.75-84.

Ishak A. and Nazar R. (2009): Laminar boundary layer flow along a stretching cylinder. - Euro. J. of Sci. Resc., vol.36, No.1, pp.22-29.

Lin H.T. and Shih Y.P. (1980): Laminar boundary layer heat transfer along static and moving cylinders. - J. Chin. Inst. Eng., vol.3, pp.73-79.

Lin H.T. and Shih Y.P. (1981): Buoyancy effects on the laminar boundary layer heat transfer along vertically moving cylinders. - J. Chin. Inst. Eng., vol.4, pp.47-51.

Wang C.Y. (2002): Flow due to a stretching boundary with partial slip - an exact solution of the Navier-Stokes equations. - Chem. Eng. Sci., vol.57, pp.3745-3747.

Xu H. and Liao S.J. (2005): Series solutions of unsteady magnetohydrodynamics flows of non-Newtonian fluids caused by an impulsively stretching plate. - J. of Non-Newtonian Fluid Mech., vol.159, pp.46-55.

Yoshimura A. and Prudhomme R.K. (1998): Wall slip corrections for Couette and parallel disc viscometers. J. Rheol., vol.32, pp.53-67.

Received: February 27, 2012

Revised: January 17, 2013 